\title{
Anthropometric Characteristics of Female and Male Athletes Bear A Different Effect on Fitness
}

\author{
Bastian Carter-Thuillier ${ }^{1,}{ }^{*}$, Rodrigo Ramírez-Campillo ${ }^{2}$, Jaime Serra-Olivares ${ }^{1}$, Francisco Gallardo ${ }^{2}$, \\ Mauricio Cresp ${ }^{1}$, Rodrigo Ojeda Nahuelcura ${ }^{1}$, Cristian Álvarez ${ }^{2}$, Cristian Martínez ${ }^{3}$ and Rodrigo \\ Cañas-Jamett ${ }^{4,5}$ \\ ${ }^{1}$ Facultad de Educación, Universidad Católica de Temuco, Temuco, Chile \\ ${ }^{2}$ Laboratorio de Rendimiento Humano, Grupo de Investigación en Calidad de Vida y Bienestar Humano, Departamento de Ciencias de la Actividad Física, Universidad de Los \\ Lagos, Osorno, Chile \\ ${ }^{3}$ Departamento de Educación Física, Deportes y Recreación, Universidad de La Frontera, Temuco, Chile \\ ${ }^{4}$ Laboratorio de Fisiología, Departamento de Ciencias Biológicas, Facultad de Ciencias de la Vida, Universidad Andres Bello, Viña del Mar, Chile \\ ${ }^{5}$ Centro de Investigación en Entrenamiento, Deporte y Salud (CEDS), Viña del Mar, Chile \\ "Corresponding author: Facultad de Educación, Universidad Católica de Temuco, Casilla D 115, Temuco, Chile. Tel: +56-452553780, Email: bcarter@uct.cl
}

Received 2018 January 11; Accepted 2019 March 15.

\begin{abstract}
Background: Anthropometric variables are highly related to physical performance, being used for the control and monitoring of athletes in different sports. However, whether anthropometric characteristics of female and male athletes bear a different effect on fitness must be determined.

Objectives: To relate anthropometric characteristics and fitness of female in comparison with male athletes.

Methods: College athletes ( $\mathrm{n}=189,112$ males) were assessed in weight, height, body mass index, lower and upper body power, agility, and endurance.

Results: Males had greater weight and height than female athletes (17 kg and $17 \mathrm{~cm}$, respectively), as well as fitness performance in all tests. In both males and females upper body power was positively correlated with body mass and height $(\mathrm{P}<0.05)$. In addition, agility was negatively correlated $(\mathrm{P}<0.05)$ with height in males and body mass in females. Aerobic fitness show a negative correlation $(\mathrm{P}<0.05)$ with body mass in males.

Conclusions: Anthropometric characteristics of female and male athletes bear a different effect on fitness.
\end{abstract}

Keywords: Sport, Field Test, Anthropometry, Gender

\section{Background}

Field tests of physical performance and anthropometry are routinely used by sport coaches and scientists, in both female (1) and male athletes (2). Although when compared to laboratory test might provide limited physiological data, field tests implicate a reduced economical cost, reduced time of application (3), and have shown a high validity, sensitivity, and reliability (4).

Assessment based on field tests, among many other uses, can be used for talent identification (5), monitoring career progression (6), ranking athletes according to competitive abilities (2), establishment of normative data regarding physical performance and anthropometric standards (7) to monitor training adaptations and prevent injuries (8). In this line, working with anthropometric variables is an affordable alternative and more practical than other forms of high-tech assessment (e.g. DXA). This may allow acquisition of important information that can be used to analyze the size, proportionality, and body composition of athletes (9).

Some reports had shown that anthropometric variables are related with speed (10), strength (11), agility (12), and endurance (13). This relation has been observed in male and female athletes from both collective (14) and individual sports (15). Also, anthropometric variables can predict the effect of age, maturation, and other biological aspects of young athletes' growth on performance (16).

Although there are reference values for body composition and anthropometry according to sport specialty and competitive level (17), relatively little information is available regarding how anthropometric variables in athletes might be related to physical performance depending on sex, which may aid to monitor training regimens (9). In addition, previous studies have included relatively little sam- 
ples of athletes, only specific athletic disciplines or athlete genders, and usually included only young athletes (18).

\section{Objectives}

The aim of this study was to establish the relationship and differences between anthropometry and physical performance of a meaningful sample of high-level male and female college athletes from different collective and individual sports. We hypothesized that anthropometry and physical performance will be related in male and female athletes, although with significant differences according to sex.

\section{Methods}

\subsection{Experimental Approach to the Problem}

A sample of college male and female athletes (characteristics in Table 1), competing in several sports (i.e. track and field, basketball, tennis, table tennis, weightlifting, soccer, rugby, handball, volleyball, field hockey, cycling, gymnastics), participated in several anthropometric and physical performance test measurements.

\subsection{Subjects}

Athletes fulfilled the following inclusion criteria (i) more than one year of competition experience in their athletic specialty, (ii) continuous training in the past 6 months, (iii) free of musculoskeletal injuries in the past 3 months, and (iv) completion of all the measurement protocols. According to these criteria, a total of 112 males (age $=20.1 \pm 2.4)$ and 77 female $($ age $=21.3 \pm 2.7$ ) were included in the study.

The institutional review board approval for our study was obtained and all athletes were carefully informed about the experiment procedures and about the possible risk and benefits associated with participation in the study. In this sense, an appropriate signed informed consent document was obtained pursuant to law before any of the tests were performed. The study was conducted in accordance with the Declaration of Helsinki.

Measurements were completed in late summer, during the beginning of the athletes' competition period. Subjects followed a familiarization period before testing to reduce any learning effects. Standardized tests were scheduled 48 hours after a competition or hard physical training and were completed in the same order (indicated below) and at the same time of the day for all athletes. Participants were motivated to give their maximum effort during performance measurements. Anthropometric measurements were completed between 10:00 -11:00, whereas performance measurements were completed between 11:00 and 16:00, always in the same location and weather condition (room temperature and humidity). Subjects were instructed to have a good night of sleep before each testing day and were asked to avoid drinking or eating at least 2 - 3 hours before measurements, including caffeinecontaining beverages, alcohol and drugs that may affect anthropometric outcomes.

\subsubsection{Anthropometric Characteristics}

To assure reliability all anthropometric measurements were completed according to the international guidelines of the ISAK (19). Height was measured to the nearest $0.1 \mathrm{~cm}$ using a calibrated wall-mounted stadiometer (Bodymeter 206, SECA, Germany), as in previous research (20). Body mass was measured to the nearest $0.1 \mathrm{~kg}$ using a calibrated digital scale (BC-554 Ironman Body Composition Monitor; Tanita, IL, USA).

\subsubsection{Vertical Jump Lower-Body Power Tests}

Testing included the execution of maximal countermovement jump (CMJ), countermovement jump with arms (CMJA), and drop jumps from 20-cm (DJ). All jumps were performed on a mobile contact mat (Globus, Codogne, Italy) with arms akimbo (except for the CMJA, for which arm swing was allowed). Take-off and landing was standardized to full knee and ankle extension on the same spot. The participants were instructed to maximize jump height and minimize ground contact time during the DJ.

\subsubsection{Medicine Ball Throwing Upper-Body Power Test (MBT)}

For this test a $2 \mathrm{~kg}$ and a $3 \mathrm{~kg}$ medicine ball was used for female and male athletes, respectively, according to previous recommendations (20). Briefly, athletes stood with the posterior trunk and head region positioned against a wall and held the ball to the front with both hands and then they were instructed to throw the medicine ball as far and fast as possible.

\subsubsection{Change of Direction Speed Time Test (CODS)}

To assess agility performance, the CODS test(i.e. Illinois agility test) was applied. Time was measured to the nearest 0.01 second using single beam infrared red photoelectric cells (Ergotester, Globus, Codogne, Italy). Start was given by a random sound that triggers timing. Athletes started supine and completed a circuit with several changes of directions, as previously described (20). The photoelectric signal was positioned at the end of the circuit and set approximately $0.7 \mathrm{~m}$ above the floor (i.e., hip level) to capture the trunk movement to avoid a false trigger from a limb. 


\subsubsection{0-m Multi Stage Shuttle Run Endurance Test (MST)}

The MST was conducted as previously described (20). Briefly, athletes ran back and forth between two lines, spaced 20-m apart, in time with the "beep" sounds from a compact disc. Each successful run of the 20-m distance was a completion of a shuttle. The beep sounded at a progressively increasing pace with every minute of the test, and the athlete had to increase speed accordingly. The athlete was warned if he did not reach the end line in time once. The test was terminated when the examinee (a) could not follow the set pace of the beeps for two successive shuttles or (b) stopped voluntarily. The scores were expressed as the last minute that the athlete completed.

At least two minutes of rest was allowed between each physical performance trial, to reduce the effects of fatigue. While waiting, participants performed low intensity activity to maintain physiological readiness for the next test. The best score of three trials was recorded for all performance tests, apart from the single MST.

\subsection{Statistical Analyses}

Data is shown as mean and standard deviation (SD). The Kolgomorov-Smirnov test and the Levene test for the assumption of normality and homogeneity of variance were used, respectively. The Pearson r correlation test was conducted to analyze the relationship between anthropometric and physical performance variables depending on sex. Sex differences for anthropometric and physical performance were analyzed using the Student $t$-test for independent samples. The statistical software SPSS (V. 20.0, 2012, United States) was used, with a 95\% confidence interval set throughout the process. The $\alpha$ level was set at $\mathrm{P}<$ 0.05. In addition, data was also assessed for meaningful relevance using an approach based on Cohen's d effect size (ES), with threshold values set at $0.20,0.60,1.2$, and 2.0 for small, moderate, large, and very large ES, respectively (21).

\section{Results}

Descriptive analysis and sex differences in anthropometric and physical performance variables are indicated in Table 1.

As expected, male athletes were heavier (i.e. $17 \mathrm{~kg}$ ) and taller (i.e. $17 \mathrm{~cm}$ ) than female athletes (Table 1).

Regarding physical performance, compared to females, male athletes had greater vertical jump performance in countermovement jump $(\sim 10 \mathrm{~cm})$, drop jump $(\sim 14 \mathrm{~cm})$ and a large greater $(\mathrm{ES}=1.14)$ vertical jump performance in countermovement jump with arms $(\sim 9 \mathrm{~cm})$. Regarding drop jump contact time, both male and females exhibit similar performance (Table 1 ).
Regarding upper body power, both male and female athletes achieved similar medicine ball throwing performance, although males showed a small meaningful greater performance $(\mathrm{ES}=0.38$ ).

In the multi stage shuttle run test male athletes exhibit greater ( $\sim 4$ minutes) performance compared to female athletes (Table 1 ).

In the change of direction speed time test, both male and female athletes achieved similar performance, although males showed a small meaningful greater performance (ES =-0.22).

In males, medicine ball throwing was positively correlated with body mass $(\mathrm{r}=0.35 ; \mathrm{P}=0.016)$ and height $(\mathrm{r}=$ $0.57 ; \mathrm{P}=0.000$ ), while performance in the multi stage shuttle run test was negatively correlated with body mass ( $\mathrm{r}=$ $-0.34 ; \mathrm{P}=0.009)$. Also, performance in the change of direction speed time test was negatively correlated with height $(\mathrm{r}=-0.27 ; \mathrm{P}=0.038)$.

In females, medicine ball throwing was positively correlated with body mass $(\mathrm{r}=0.40 ; \mathrm{P}=0.000)$ and height $(\mathrm{r}$ $=0.27 ; \mathrm{P}=0.016)$. Also, performance in the change of direction speed time test was negatively correlated with body mass $(r=-0.29 ; P=0.026)$.

\section{Discussion}

The aim of this study was to establish the relationship and differences between anthropometry and physical performance of a meaningful sample of high-level male and female college athletes from different collective and individual sports. According to our hypothesis, anthropometry and physical performance were related in male and female athletes, although with significant differences according to sex.

Among our main results, a correlation was observed between height and agility performance in males (i.e. taller athletes showed lower agility performance). Also, our results indicate a correlation between body mass and agility in female athletes (i.e. heavier athletes were less agile). In the case of males, the correlation may be explained as an athlete with lower center of gravity might apply horizontal force more quickly than a taller athlete (due to less time required to lower their center of gravity in preparation for a lateral direction), meaning that a faster change of direction would be possible (22). In the case of females, the correlation may be explained by the effect of a greater mass on inertia during change of direction movements (23). Because very little research has attempted to correlate anthropometric variables and change of direction speed performance, our results may be considered novel. The present results corroborate the theoretical model proposed by Sheppard and Young (22), where anthropometry 


\begin{tabular}{|c|c|c|c|c|c|}
\hline & $\operatorname{Males}(\mathrm{N}=112)^{\mathrm{a}}$ & Females $(N=77)^{a}$ & $\mathbf{F}$ & $P(\text { Student } t \text {-test })^{b}$ & Cohen's d ES \\
\hline Body mass, kg & $79.0 \pm 11.9$ & $62.4 \pm 11.1$ & 0.78 & 0.001 & 1.43 \\
\hline Height, cm & $177.7 \pm 7.8$ & $160.7 \pm 7.0$ & 1.98 & 0.001 & 2.30 \\
\hline Countermovement, cm & $34.3 \pm 9.0$ & $24.6 \pm 5.9$ & 3.75 & 0.001 & 1.27 \\
\hline Countermovement jump with arms, $\mathrm{cm}$ & $41.3 \pm 8.7$ & $32.6 \pm 6.3$ & 0.91 & 0.063 & 1.14 \\
\hline Drop jump height, cm & $39.8 \pm 8.7$ & $25.9 \pm 6.5$ & 4.18 & 0.001 & 1.81 \\
\hline Drop jump contact time, ms & $410 \pm 128$ & $390 \pm 146$ & 0.32 & 0.371 & 0.14 \\
\hline Medicine ball throwing, $\mathrm{m}$ & $6.4 \pm 0.7$ & $6.1 \pm 1.0$ & 3.28 & 0.035 & 0.38 \\
\hline Multi stage shuttle run test, min & $9.1 \pm 2.3$ & $5.0 \pm 2.4$ & 0.04 & 0.001 & 1.75 \\
\hline Change of direction speed time test, $s$ & $16.7 \pm 1.9$ & $17.6 \pm 5.8$ & 17.6 & 0.166 & -0.22 \\
\hline
\end{tabular}

${ }^{\mathrm{a}}$ Values are expressed as mean $\pm \mathrm{SD}$.

${ }^{\mathrm{b}}$ Values represent comparisons between males and females.

was identified as a key agility component. Interestingly, no significant difference $(\mathrm{P}=0.166)$ in agility performance was observed between female (17.6 s) and male (16.7 s) athletes. The fact that female athletes were shorter than males (161 cm compared to $178 \mathrm{~cm}$, respectively) might help explain these results, because, as explained previously, a lower center of gravity in the case of females might have helped them to apply horizontal force more quickly than taller athletes (i.e., males) (22).

Medicine ball throwing was correlated with body mass and height in both male and female athletes. It is possible that a higher body mass was related with higher absolute muscle mass, thus allowing better throwing performance (24). In fact, male athletes, who had significantly greater body mass than females, showed greater throwing performance, even considering that males used a 50\% heavier medicine ball during testing.

Endurance running performance has a structural basis (25). In this line, our results revealed that males showed a negative correlation between multi stage shuttle run test performance and body mass. However, male athletes were heavier than female athletes, but the former showed higher performance in the multi stage shuttle run test. Thus, aside from body mass, other's factors may affect MST performance. In fact, it has been stated that successful middle and long-distance runners may require not only optimal cardiovascular capabilities but also neuromuscular characteristics related to voluntary and reflex neural activation, muscle force and elasticity, running mechanics, and anaerobic characteristics (26). In this sense, male athletes, in spite of greater body mass than female athletes, due to their greater neuromuscular capabilities (i.e., higher CMJ, drop jump), might be able to develop greater performance in the MST, an endurance test which requires repeated sudden changes of directions, with a greater demand on the muscle's stretch-shortening cycle capabilities (27).
Although jump performance was not related to anthropometry, male athletes had 21\% greater body mass and $22 \%$ jumping capability than female athletes. Considering that greater jumping performance is associated to greater muscle power (28) and because greater muscle power is associated with greater muscle mass, it might be speculated that male athletes had greater muscle mass than female athletes and this might allow the former to achieve greater muscle power production and thus greater jump performance. However, regarding drop jump contact time, both male and female athletes exhibit similar performance, suggesting that different muscle strength variables (e.g. power, reactive strength, maximal strength) might not be equally related with anthropometric variables. This deserves future investigation.

\subsection{Conclusions}

In conclusion, anthropometry and physical performance are related in male and female athletes, although with significant differences according to sex. These results should be taken into account by anthropometrists, coaches, and strength and conditioning specialists. In addition, aside from a comparative or correlation analysis, a systematic assessment follow up in athletes that incorporates variables such as height and body mass may help to identify athletes at risk of injury (8). A possible limitation of the present study was the lack of sport-specific analysis (e.g. basketball, soccer). As some sports were represented by small sample sizes, future research should consider individual sport teams with larger sample sizes. Although statistically significant findings were described by gender, caution is advised to interpret current findings, as some group sizes were small with wide standard deviations.

\section{Footnotes}

Authors' Contribution: Bastian Carter-Thuillier and Rodrigo Ramírez-Campillo contributed equally to this 
work. Study design: Bastian Carter-Thuillier, Rodrigo Ramírez-Campillo, and Mauricio Cresp-Barria; data collection: Bastian Carter-Thuillier, Rodrigo Ramírez-Campillo, Mauricio Cresp-Barria, and Francisco Gallardo-Fuentes; data collection (field test): Rodrigo Ojeda Nahuelcura; data analysis: Jaime Serra-Olivares and Cristian Álvarez; drafting of manuscript: Bastian Carter-Thuillier, Rodrigo Ramírez-Campillo, Francisco Gallardo-Fuentes, Jaime Serra-Olivares, Cristian Álvarez, Cristian Martínez and Rodrigo Cañas-Jamett; revision of manuscript: Cristian Martínez and Rodrigo Cañas-Jamett.

Conflict of Interests: Authors do not have any conflict of interest associated with the development of this research or publication of their results.

Funding/Support: It is none declared by the author.

\section{References}

1. Vescovi JD, McGuigan MR. Relationships between sprinting, agility, and jump ability in female athletes. J Sports Sci.2008;26(1):97-107. doi: 10.1080/02640410701348644. [PubMed: 17852692].

2. Nikolaidis PT, Ingebrigtsen J. Physical and physiological characteristics of elite male handball players from teams with a different ranking. J Hum Kinet. 2013;38:115-24. doi: 10.2478/hukin-2013-0051. [PubMed: 24235989]. [PubMed Central: PMC3827752]

3. Pereira LA, Flatt AA, Ramirez-Campillo R, Loturco I, Nakamura FY. Assessing shortened field-based heart-rate-variability-data acquisition in team-sport athletes. Int J Sports Physiol Perform. 2016;11(2):154-8. doi 10.1123/ijspp.2015-0038. [PubMed: 26115088].

4. Bangsbo J, Iaia FM, Krustrup P. The Yo-Yo intermittent recovery test : A useful tool for evaluation of physical performance in intermittent sports. Sports Med. 2008;38(1):37-51. doi: 10.2165/00007256 200838010-00004. [PubMed: 18081366].

5. Reilly T, Williams AM, Nevill A, Franks A. A multidisciplinary approach to talent identification in soccer. J Sports Sci. 2000;18(9):695-702. doi 10.1080/02640410050120078. [PubMed: 11043895].

6. Pyne DB, Gardner AS, Sheehan K, Hopkins WG. Fitness testing and career progression in AFL football. J Sci Med Sport. 2005;8(3):321-32. [PubMed: 16248473].

7. Gabbett TJ. Physiological and anthropometric characteristics of elite women rugby league players. J Strength Cond Res. 2007;21(3):875-81. doi: 10.1519/R-20466.1. [PubMed: 17685702].

8. Kemper GL, van der Sluis A, Brink MS, Visscher C, Frencken WG, Elferink-Gemser MT. Anthropometric injury risk factors in elitestandard youth soccer. Int JSports Med.2015;36(13):1112-7. doi:10.1055/s0035-1555778. [PubMed: 26258817].

9. Brocherie F, Girard O, Forchino F, Al Haddad H, Dos Santos GA, Millet GP. Relationships between anthropometric measures and ath letic performance, with special reference to repeated-sprint ability, in the Qatar national soccer team. J Sports Sci. 2014;32(13):1243-54. doi: 10.1080/02640414.2013.862840. [PubMed: 24742185].

10. Ozkan A, Kayihan G, Koklu Y, Ergun N, Koz M, Ersoz G, et al. The relationship between body composition, anaerobic performance and sprint ability of amputee soccer players. J Hum Kinet. 2012;35:141-6. doi: 10.2478/v10078-012-0088-3. [PubMed: 23486067]. [PubMed Central: PMC3588686].

11. Bilsborough JC, Greenway KG, Opar DA, Livingstone SG, Cordy JT, Bird SR, et al. Comparison of anthropometry, upper-body strength, and lower-body power characteristics in different levels of Australian football players. J Strength Cond Res. 2015;29(3):826-34. doi: 10.1519/JSC.0000000000000682. [PubMed: 25226309]
12. Spiteri T, Newton RU, Binetti M, Hart NH, Sheppard JM, Nimphius S. Mechanical determinants of faster change of direction and agility performance in female basketball athletes. J Strength Cond Res. 2015;29(8):2205-14. doi: 10.1519/JSC.0000000000000876. [PubMed: 25734779].

13. Haakonssen EC, Barras M, Burke LM, Jenkins DG, Martin DT. Body composition of female road and track endurance cyclists: Normative values and typical changes. Eur J Sport Sci. 2016;16(6):645-53. doi: 10.1080/17461391.2015.1084538. [PubMed: 26366462].

14. Robertson S, Woods C, Gastin P. Predicting higher selection in elite junior Australian Rules football: The influence of physical performance and anthropometric attributes. J Sci Med Sport. 2015;18(5):601-6. doi: 10.1016/j.jsams.2014.07.019. [PubMed: 25154704]

15. Bond D, Goodson L, Oxford S, Nevill A, Duncan M. The association between anthropometric variables, functional movement screen scores and $100 \mathrm{~m}$ freestyle swimming performance in youth swimmers. Sports. 2015;3(1):1-11. doi: 10.3390/sports3010001.

16. Torres-Unda J, Zarrazquin I, Gravina L, Zubero J, Seco J, Gil SM, et al. Basketball performance is related to maturity and relative age in elite adolescent players. J Strength Cond Res. 2016;30(5):1325-32. doi: 10.1519/JSC.0000000000001224. [PubMed: 26439783].

17. Santos DA, Dawson JA, Matias CN, Rocha PM, Minderico CS, Allison DB, et al. Reference values for body composition and anthropometric measurements in athletes. PLoS One. 2014;9(5). e97846. doi: 10.1371/journal.pone.0097846. [PubMed: 24830292]. [PubMed Central: PMC4022746].

18. Pion J, Segers V, Fransen J, Debuyck G, Deprez D, Haerens L, et al. Generic anthropometric and performance characteristics among elite adolescent boys in nine different sports. Eur J Sport Sci. 2015;15(5):357-66. doi: 10.1080/17461391.2014.944875. [PubMed: 25143133].

19. Marfell-Jones M, Olds T, Stewart A, Carter L. International standards for anthropometric assessment. Potchefstroom: ISAK; 2006.

20. Ramirez-Campillo R, Vergara-Pedreros $M$, Henriquez-Olguin $C$, Martinez-Salazar C, Alvarez C, Nakamura FY, et al. Effects of plyometric training on maximal-intensity exercise and endurance in male and female soccer players. J Sports Sci. 2016;34(8):687-93. doi: 10.1080/02640414.2015.1068439. [PubMed: 26197721].

21. Hopkins WG, Marshall SW, Batterham AM, Hanin J. Progressive statistics for studies in sports medicine and exercise science. Med Sci Sports Exerc. 2009;41(1):3-13. doi: 10.1249/MSS.0b013e31818cb278. [PubMed: 19092709].

22. Sheppard JM, Young WB. Agility literature review: Classifications, training and testing. J Sports Sci. 2006;24(9):919-32. doi: 10.1080/02640410500457109. [PubMed:16882626].

23. Izquierdo M. Biomechanics and neuromuscular bases of physical activity and sport. Madrid: Panamericana; 2008.

24. Kyriazis T, Terzis G, Karampatsos G, Kavouras S, Georgiadis G. Body composition and performance in shot put athletes at preseason and at competition. Int J Sports Physiol Perform. 2010;5(3):417-21. [PubMed: 20861531].

25. Weyand PG, Davis JA. Running performance has a structural basis. J Exp Biol. 2005;208(Pt 14):2625-31. doi: 10.1242/jeb.01609. [PubMed: 16000532].

26. Noakes TD. Implications of exercise testing for prediction of athletic performance: A contemporary perspective. Med Sci Sports Exerc. 1988;20(4):319-30. [PubMed: 3050352].

27. Buchheit M. The 30-15 intermittent fitness test: Accuracy for individualizing interval training of young intermittent sport players. J Strength Cond Res. 2008;22(2):365-74. doi: 10.1519/JSC.ob013e3181635b2e. [PubMed: 18550949].

28. Markovic G, Mikulic P. Neuro-musculoskeletal and performance adaptations to lower-extremity plyometric training. Sports Med. 2010;40(10):859-95. doi: 10.2165/11318370-000000000-00000. [PubMed: 20836583]. 